\section{Bohr Effect Explained}

Tue affinity of haemoglobin for oxygen depends on the $p \mathrm{H}$ of the environment, and this is physiologically important because as the concentrations of carbon dioxide and therefore the need for oxygen increase in tissues, the $p \mathrm{H}$ decreases and so does the affinity of haemoglobin for oxygen. In other words, the higher the concentration of carbon dioxide, the easier it is to transfer oxygen from haemoglobin to a tissue. This Bohr effect must depend on the state of ionization of a few amino-acid residues in the two pairs of interlocking protein chains that constitute a haemoglobin molecule, for only the charge of a protein depends on the $p \mathrm{H}$ of its milieu.

But which residues are involved in the Bohr effect? On page 1240 of this issue, Perutz's group suggests an answer which takes into account all the apparently conflicting chemical evidence accumulated in the past thirty years. It believes that the imidazole sidechains of the histidine residues at the end of one pair of chains (the $\beta$ chains) together with the free amino groups at the opposite end of the other pair of chains (the $\alpha$ chains) are essentially responsible for the Bohr effect.

At various times in the past, three types of ionizing groups in haemoglobin have been suggested by biochemists as candidates for producing the Bohr effect: sulphydryl groups of cysteinc residues, the basic imidazole side-chains of histidine residues and the terminal amino groups of the first amino-acid of the four chains. Perutz's group has shown by X-ray crystallography that during the oxygenation-deoxygenation cycle, the molecule changes its shape and the imidazole group of the last histidine residue in the two $\beta$ chains is involved. In oxyhaemoglobin, these residues are free for ionization, but in deoxyhaemoglobin they interact with carboxylate groups of aspartic acid residues further in the $\beta$ chain. Perutz's group also finds that, in the $\alpha$ chains, a similar sort of interaction occurs when oxyhaemoglobin loses its oxygen. The frec amino groups at the end of one $\alpha$ chain interact with the free corboxy group at the opposite end of its twin, so that the two a chains are linked head to tail. Both these interactions, which take place on deoxygenation, will increase the affinity of basic groups in the molecule for hydrogen ions and decrease the affinity for oxygen.

Another result of the conformational change on deoxygenation is the partial sequestration of one of the sulphydryl groups in the $\beta$ chains. When this $\mathrm{SH}$ group is blocked by chemical reaction with a blocking agent, not only is the conformational change on deoxygenation prevented but also a part of the Bohr effect is lost. Perutz and his colleagues have studicd this chemically modified form of oxyhaemoglobin, and the chemically modified form of deoxyhaemoglobin obtaincd from it. First, they proved that the $\mathrm{SH}$ group blocking agent itself does not cause any detectable change in the conformation of oxyhaemoglobin. They then have determined which of the amino-acid residues has an altered position in deoxyhaemoglobin with a blocked SH group. It turns out that in this chemically modified form of deoxyhaemoglobin the conformation of the histidine residue at the very end of the two $\beta$ chains is restrictcd compared with unmodified deoxyhaemoglobin. This appears to be the only residue to be affected and so they argue that this is the residue that must be responsible for that portion. of the Bohr effect that is inhibited by $\mathrm{SH}$ reagents. The erystallographic evidence therefore shows that oxygenation-deoxygenation involves interaction between the ends of the a chains and change in the geometry of the terminal histidine residues of the $\beta$ chains. Kilmartin and Rossi-Bernardi (see page 1243) have also shown that chemically blocking the amino groups of the a chains reduces the Bohr effect by about 25 per cent. 'Thus the crystallography provides at long last a molecular understanding of the Bohr effect and several other of the chemical properties of haemoglobin into the bargain.

\section{LINNEAN SOCIETY \\ Bringing back Bones}

THE four palaeontologists who returned last November from their search for mammal ancestors in Lesotho are obviously not going to sit on their findings for long. Two members of the expedition, which was sponsored by the Natural Environment Research Council, gave a preliminary report at the meeting of the Iinnean Society on June 19. Their finds in the Red Beds of western Lesotho included remains of the mammal-like reptiles which were the immediate precursors of the first mammals, some dinosaurs and the complete

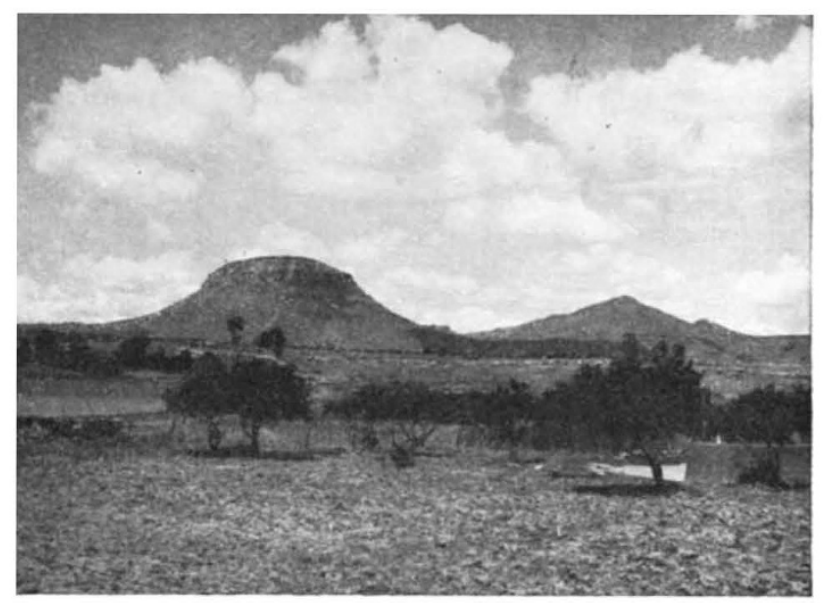

A view of the area in western Lesotho where some of the fossils were found, including the complete skull of what is probably a very early mammal. 